\title{
The effect of urine pregnancy testing on timing of accessing antenatal care and abortion services in Western Nigeria
}

\author{
Mustafa Adelaja Lamina \\ Maternal and Fetal Health Research Unit, Department of Obstetrics and Gynaecology, Olabisi Onabanjo University Teaching Hospi- \\ tal, Lagos, Nigeria \\ Email: ademustapha_2003@yahoo.co.uk
}

Received 16 May 2013; revised 18 June 2013; accepted 26 June 2013

Copyright (C) 2013 Mustafa Adelaja Lamina. This is an open access article distributed under the Creative Commons Attribution License, which permits unrestricted use, distribution, and reproduction in any medium, provided the original work is properly cited.

\begin{abstract}
Objective: Effective confirmation of pregnancy is a basic component of reproductive health services. It is a determinant for accessing antenatal care (ANC) if the pregnancy is wanted and abortion services if the pregnancy is unwanted. This study examined the effect of urine pregnancy testing in the timing of presentation for pregnancy care. Method: A cross-sectional study was conducted among 320 women presenting for antenatal care and 300 women presenting for abortion at private sector clinics in Western Nigeria. Results: The median age at first presentation was 20 weeks for ANC clients and 8 weeks for abortion clients. Obtaining urine pregnancy test of one's accord was associated with a decrease in the gestational age at presentation of 3.7 weeks and 1.5 weeks for antenatal and abortion clients, respectively, independent of all other factors. Conclusion: Bearing in mind that the clinical benefit and public health impact of early presentation for antenatal and abortion services are unequivocal, strategies aimed at decreasing gestational age at presentation for pregnancy care should be given priority. "Fast-track" urine pregnancy testing services should be promoted in the private and public clinics in Nigeria.
\end{abstract}

Keywords: Urine Pregnancy Testing; Timing; Accessing; Antenatal; Abortion

\section{BACKGROUND}

Effective confirmation of pregnancy is a basic component of reproductive health services [1-3]. It is a prerequisite for accessing antenatal care (ANC) if the pregnancy is wanted and abortion services if pregnancy is unwanted. Pregnancy test is also often required to rule out pregnancy prior to contraception [4]. While blood tests, clinical examination and ultrasound scan may be used, administration of sensitive beta-human chorionic gonadotrophin $(\beta$-hCG) urine pregnancy test is an effective, non-invasive and low-cost means of early pregnancy confirmation that is feasible in many low-resource settings $[4,5]$.

There is a general belief that urine pregnancy testing, though theoretically available at many public and private health facilities in Nigeria, is under-utilized by clinic staff. Lack of early pregnancy confirmation services may contribute to late presentation for antenatal care and abortion services, which is the norm in Nigeria and many other parts of sub-Saharan Africa $[2,6,7]$.

The importance of early pregnancy detection is wellknown but little is known about how the availability and accessibility of early pregnancy confirmation services, in the form of urine pregnancy testing, may affect gestational age at presentation for ANC and abortion services. Therefore, the objective of this study is to examine the role of urine pregnancy testing in the timing of presentation for private sector ANC and abortion services in Western Nigeria.

\section{METHODS}

This study was carried out in Ogun State, which is one of the thirty-six states in Nigeria and is located in the Western region of the country. Ogun state is divided into four geopolitical areas: Egba, Yewa, Ijebu and Remo. Health services are provided by both public and private health facilities. Full payment is made for health services obtained from private health facilities while health services in the public sector are subsidized. Abortion law is restrictive in Nigeria. Abortion services are not liberally provided in public hospitals and such services are offered to clients on the ground that their life is threatened. Hence, abortion services are clandestinely provided by private health facilities (hospitals and clinics) and some 
other quacks like pharmacists, chemists, nurses and traditional healers.

Very few private hospitals and clinics participate in the provision of abortion services. There were more private hospitals and clinics in the two urban than the two other semi-urban areas in the provision of abortion services. Hence, two private hospitals each were randomly selected from the two urban areas and one each from the two semi-urban areas making a total of six private hospitals taking part in the study. All the private hospitals provide antenatal care services for both "low-risk" and "high-risk" obstetric patients because majority of them are manned by doctors/gynaecologists.

A cross-sectional prospective study was undertaken in two groups of pregnant women attending four of the private hospitals in two urban areas, and two of the private hospitals in two semi-urban areas: women making their first visit for either 1) ANC services or 2) first or second trimester abortion services.

Over a twelve-week period from September to November 2011), consecutive consenting women, who were aged 14 years or older, at all of the facilities providing these services in the study areas were interviewed and asked questions on timing, method and location of pregnancy confirmation, and on referral pathways to current point of service. Refusals were limited $(<4 \%)$. Participants were interviewed either in English or their native (home) language by using a standardized, pre-tested questionnaire. In accordance with the practice in the private hospitals in this setting, the gestational age was determined by using information from menstrual history and clinical examination for the ANC attendees whereas for abortion clients, further assessment of gestational age with ultrasound scan was done in addition to menstrual history and clinical examination.

In addition, in-person interviews with health care providers using a structured instrument were conducted at eight randomly selected private clinics in the four areas. A telephonic survey was also conducted among providers in a random sample of $50 \%$ of private clinics in the four areas. In all 96 providers (30 professional nurses, 50 auxiliary nurses and 16 community health extension workers and assistants) were interviewed. The provider surveys collected basic information about the availability and provision of pregnancy testing services at the clinics, as well as unprompted information about provider's opinions of the merits and demerits of offering urine pregnancy testing in private sector clinics.

Sample size calculations were done for one outcome: the change in gestational age at first visit associated with obtaining a urine pregnancy test. The calculation was based on the following assumptions: 1) an average gestational age at first visit among all women of 21 weeks for ANC clients and 9 weeks for abortion clients, 2) a mini- mum clinically relevant change in gestational age at first visit due to obtaining a urine pregnancy test of two weeks, 3) at least one-third of subjects having obtained a urine pregnancy test, and 4) a two-sided alpha of 0.05 with $80 \%$ power to detect the difference. Under these assumptions, 222 women attending for ANC and 222 women attending for abortion were required. This sample was exceeded in both groups.

The data was analyzed by using the statistical programme SPSS 16.0 for Windows. Bivariate analyses employed Wilcoxon rank sum and $\chi$-square. Separate multiple linear regression models were developed to examine how demographic, behavioural, and health service-related factors were associated with gestational age at presentation for ANC and abortion services.

Written informed consent was obtained from all participants and the approval to conduct the study was granted by the Ethics Review Committee of Olabisi Onabanjo University Teaching Hospital, Sagamu, Ogun State, Nigeria.

\section{RESULTS}

\subsection{Socio-Demographic Characteristics}

A total of 620 women participated in the study: 320 at first ANC visit while 300 were presenting for first and second trimester abortion. The participants were young women (median age 27 years; range 14 - 45). Ten percent of ANC clients $(n=32)$ and $15 \%$ of the abortion clients $(n=45)$ were teenagers. Most participants had had previous pregnancies $(n=415,67 \%)$, were currently in relationship $(\mathrm{n}=434,70 \%)$. Women seeking abortion were more likely to be unmarried ( $49 \%$ vs $11 \%$, Odds ratio 7.8 , CI $95 \%)$, students $(32 \%$ vs $8 \%$, Odds ratio 5.4 , CI 95\%) and Christians (73\% vs 51\%, Odds ratio 2.6, CI 95\%) than women presenting for ANC.

\subsection{Knowledge of Pregnancy Testing and Timing of Presentation for Care}

Overall, about half of the women spontaneously mentioned urine pregnancy testing as a way for woman to detect a pregnancy, but almost all had heard of urine pregnancy testing when asked directly (Table 1).

However, most women did not know that a urine pregnancy test could detect a pregnancy at about two weeks after a missed period. About half of the women thought that a urine pregnancy test could only be used to detect pregnancy greater than two months after a missed period.

Women's knowledge of the appropriate time in pregnancy to make the first health service visit was low: $76 \%$ $(\mathrm{n}=243)$ in the ANC sample and $34 \%(\mathrm{n}=102)$ in the abortion sample thought the first visit should be made at three months of pregnancy or later (Table 1). 
Table 1. Knowledge and pattern of urine pregnancy testing among private sector ANC and abortion clients in Western Nigeria.

\begin{tabular}{|c|c|c|}
\hline Characteristics & $A N C n=320(\%)$ & Abortion clients $n=300(\%)$ \\
\hline $\begin{array}{l}\text { Spontaneously mentioned urine pregnancy testing as a way for a } \\
\text { woman to detect her pregnancy/know she might be pregnant }\end{array}$ & $166(52)$ & $162(54)$ \\
\hline Heard of urine pregnancy testing when asked directly & $304(95)$ & $285(95)$ \\
\hline \multicolumn{3}{|c|}{ How long after missed period before a urine pregnancy test can detect pregnancy } \\
\hline 1 - 2 weeks & $29(9)$ & $54(18)$ \\
\hline 1 month & $93(29)$ & $66(22)$ \\
\hline 2 months & $112(35)$ & $96(32)$ \\
\hline 3 months & $48(15)$ & $48(16)$ \\
\hline Don’t know & $38(12)$ & $36(12)$ \\
\hline Willing to pay for urine pregnancy test at a private clinic & $224(70)$ & $63(21)$ \\
\hline \multicolumn{3}{|c|}{ Appropriate time after fallen pregnant to make first visit for pregnancy care } \\
\hline Immediately or as soon as pregnancy is detected & $32(10)$ & $63(21)$ \\
\hline$\geq 1$ month & $16(5)$ & $45(15)$ \\
\hline$\geq 2$ months & $29(9)$ & $90(30)$ \\
\hline$\geq 3$ months & $80(25)$ & $60(20)$ \\
\hline$\geq 4$ months & $64(20)$ & $33(11)$ \\
\hline$\geq 5$ months & $80(25)$ & $9(3)$ \\
\hline$\geq 6$ months & $19(6)$ & $0(0)$ \\
\hline Urine pregnancy testing obtained from pharmacy in this pregnancy & $93(29)$ & $117(39)$ \\
\hline $\begin{array}{l}\text { Sent from a private sector clinic to pharmacy to obtain pregnancy } \\
\text { test and return to clinic with result }\end{array}$ & $51(16)$ & $111(38)$ \\
\hline
\end{tabular}

\subsubsection{Characteristics of Current Pregnancy}

Median gestational age at first presentation was 20 weeks (inter-quartile range (IQR) 16-24) for ANC clients. Only $25(7.8 \%)$ of the ANC clients presented in the first trimester. Median gestational age at presentation for abortion clients was 8 weeks (IQR 6-12).

Majority of the participants (ANC; $\mathrm{n}=182,57 \%$, abortion; $\mathrm{n}=183,61 \%$ ) had confirmation of pregnancy and first pregnancy-related visit done outside the private health services (private pharmacies). ANC clients attended a median of 2 other health facilities before presenting at the current private clinic to initiate pregnancy care, while abortion clients attended a median of 3 other facilities. Twenty-nine percent $(\mathrm{n}=93)$, and 39\% $(\mathrm{n}=$ 117) of ANC and abortion clients respectively, on their own obtained a urine pregnancy test at a private pharmacy during this pregnancy. In addition, many participants reported that during this pregnancy, they had been sent from a private clinic to procure a pregnancy test at a pharmacy and then return with the results before care would be initiated (ANC clients: $\mathrm{n}=51,16 \%$, abortion clients: $\mathrm{n}=111,37 \%)$. Importantly, providers reported that pregnancy tests were available in all of the clinics where women reported that this practice took place.

\subsubsection{Pregnancy Testing and Gestational Age at Presentation}

In bivariate analysis, obtaining a urine pregnancy test of one's own accord at a private pharmacy was associated with a 5-week and 2-week decrease in gestational age at presentation for ANC and abortion clients, respectively. On the other hand, being sent from a private clinic to have a pregnancy test at a private pharmacy and return with the results was associated with 3-week increase in gestational age at presentation among ANC clients and 1.5 -week increase in gestational age at presentation among abortion clients.

In multivariate analysis, several factors were shown to be associated with gestational age at first presentation to a private clinic for pregnancy-care, and these factors were similar across the two samples of pregnant women (Table 2). Obtaining a pregnancy test of one's own ac- 
Table 2. Independent predictors from multiple linear regression models of gestational age at booking among private sector ANC and Abortion clients in Western Nigeria.

\begin{tabular}{|c|c|c|c|}
\hline ANC clients ${ }^{*}$ Characteristic & Decrease in number of weeks $95 \%$ CI & p-value & gestational age for ANC \\
\hline \multicolumn{4}{|c|}{ Obtaining a urine pregnancy test at a private pharmacy } \\
\hline No & (Reference category) & & \\
\hline Yes & -3.5 weeks & $-5.8 ;-1.2$ & 0.01 \\
\hline \multicolumn{4}{|l|}{ Age } \\
\hline$<20$ & (Ref) & & \\
\hline $20+$ & -2.7 weeks & $-5.3 ;-0.1$ & 0.05 \\
\hline \multicolumn{4}{|c|}{ Main language spoken } \\
\hline Hausa & (Ref) & & \\
\hline Yoruba & -3.5 weeks & $-5.8 ;-1.2$ & 0.01 \\
\hline Igbo & -2.8 weeks & $-5.4 ;-0.2$ & 0.05 \\
\hline \multirow[t]{2}{*}{ English } & -5.4 weeks & $-9.1 ;-1.7$ & 0.01 \\
\hline & \multicolumn{3}{|c|}{ Sent from private clinic to obtain pregnancy test at private pharmacy } \\
\hline Yes & (Ref) & & \\
\hline No & -2.7 weeks & $-5.3 ;-0.1$ & 0.05 \\
\hline TOP clients ${ }^{* *}$ Characteristics & Decrease in number of weeks $95 \%$ CI & p-value & gestational age for abortion \\
\hline \multicolumn{4}{|c|}{ Obtaining a urine pregnancy test at a private pharmacy } \\
\hline No & (Ref) & & \\
\hline Yes & -1.5 weeks & $-2.9 ;-0.04$ & 0.04 \\
\hline \multicolumn{4}{|c|}{ Number of previous pregnancies } \\
\hline 0 & (Ref) & & \\
\hline $1+$ & -0.5 weeks & $-1.0 ;-0.4$ & 0.04 \\
\hline \multicolumn{4}{|c|}{ Main language spoken } \\
\hline Hausa & (Ref) & & \\
\hline Yoruba & -1.5 weeks & $-2.9 ;-0.04$ & 0.04 \\
\hline Igbo & +0.7 weeks & $-1.2 ;+2.6$ & 0.6 \\
\hline English & -2.4 weeks & $-4.5 ;-0.3$ & 0.01 \\
\hline \multicolumn{4}{|c|}{ Sent from private clinic to obtain pregnancy test at Private pharmacy } \\
\hline Yes & (Ref) & & \\
\hline No & -1.2 & $-2.4 ;-0.2$ & 0.03 \\
\hline
\end{tabular}

*Adjusted for language, level of education, employment status, source of financial support, prior pregnancy, and knowledge of other reproductive health services; ${ }^{* *}$ Adjusted for language, level of education, employment status, source of financial support, prior pregnancy, and knowledge of other reproductive health services.

cord from a private pharmacy was associated with a 3.5week decrease in the gestational age at ANC presentation and a 1.5-week decrease in gestational age at presentation for an abortion. Being sent from any clinic to obtain pregnancy test at a private pharmacy and return with the results increased gestational age at presentation by 2.7 weeks among ANC clients and 1.2 weeks among abortion clients. Women older than 20 years booked significantly earlier for both ANC and abortion services than teenagers, and for both ANC and abortion services, 
Hausa speakers booked significantly later than women speaking Yoruba, Igbo or English.

\subsubsection{Providers' Knowledge and Attitude as Regards Urine Pregnancy Testing Services}

The majority of the 96 private healthcare providers interviewed $(\mathrm{n}=86,90 \%)$ reported that pregnancy tests were available at their clinics. However, less than half of the providers $(\mathrm{n}=39,45 \%)$ where pregnancy testing is available said that there were no written policy or guidelines for their clinics. Less than half of the providers had ever been trained on when $(n=43,45 \%)$ or how $(n=36$, $38 \%)$ to do a urine pregnancy test. Just over half $(\mathrm{n}=53$, $55 \%$ ) thought that they should always give a pregnancy test if a client requested one. Forty-eight percent $(n=46)$ thought they should not do tests on teenagers because "they should not be sexually active or using contraception whatsoever". Majority of providers $(n=72,75 \%)$ thought that pregnancy tests should be available as part of routine care at private sector clinics. However, fewer $(\mathrm{n}=62,65 \%)$ felt that it should be made very cheap. All providers said that there are advantages to promoting pregnancy testing. Seventy-five percent $(n=72)$ said it would definitely facilitate earlier ANC and abortion presentation and $20 \%(\mathrm{n}=19)$ said it would provide an opportunity to educate women about pregnancy and pregnancy prevention. Sixty percent $(n=58)$ said that there were also disadvantages. The main disadvantages mentioned were: staff shortages $(n=24,25 \%)$, a concern that pregnancy testing would promote "irresponsible client behaviour" and result in decreased contraceptive use ( $\mathrm{n}=38,40 \%)$, and the possibility of clients "abuse" of the pregnancy testing service if it is made cheap $(\mathrm{n}=$ $24,25 \%)$. Most providers $(\mathrm{n}=86,90 \%)$ thought that pregnancy tests should be conducted by community health workers, lay counselors, clinic assistants, or "anyone available", as opposed to nursing staff. While all the clinics had a supply of urine pregnancy tests in stock, only one clinic had a sign announcing that pregnancy tests were available.

\section{DISCUSSION}

This is one of few studies in sub-Saharan Africa to assess the relationship between access to urine pregnancy testing as a means of pregnancy confirmation and gestational age at presentation for pregnancy care $[8,9]$.This study has shown that timing of presentation is influenced by access to urine pregnancy testing. Obtaining a urine pregnancy test of one's own accord from a pharmacy is associated with a significant decrease in the gestational age at presentation for both ANC and abortion, independently of all other factors, including age, level of education, employment status, prior pregnancy, knowl- edge of other reproductive services and language.

Although urine pregnancy tests are theoretically available at most private clinics in Nigeria, including those in this study, participants who accessed a urine pregnancy test of their own accord obtained it from a private pharmacy separate from the clinic. Furthermore, an appreciable number of participants reported that during their current pregnancy, they had been sent from a private clinic to purchase a pregnancy test at a private pharmacy separate from the clinic and then return to the private clinic with the results before care could be initiated. This practice requires a review as women should be able to access urine pregnancy testing as part of routine care at private clinics where pregnancy care would be initiated in most cases. Pharmacy urine pregnancy test may be expensive for many women. In addition, this study has demonstrated that sending pregnant women who have accessed private services to private pharmacies separate from the private clinics for pregnancy confirmation via urine pregnancy testing contributes to delays in presentation. Some women may not return to health care services at all, or in the case of abortion may return too late for safe procedure. Women sent away from the clinic for confirmation of pregnancy who turn out not to be pregnant will likely not return to the clinic with negative result. This represents a missed opportunity for contraceptive or pre-conception counseling. There is a need to explore the reasons for providers' reluctance to use pregnancy tests in the private sector.

Early presentation for ANC is associated with improved health outcomes for both mother and child. These include decreased risk of pregnancy loss, decreased maternal morbidity and mortality, and decreased neonatal morbidity and mortality [2]. For ANC to be fully effecttive, the initial visit should take place as soon as the pregnancy is suspected and certainly before 12 weeks of pregnancy [2]. Similarly, abortions that take place during the first 12 weeks of pregnancy and certainly before 10 weeks, are safer and more cost-effective than second trimester abortions $[3,10]$.

Obviously, if most pregnant women make their first visit for ANC and abortion at 20 weeks and 12 weeks respectively, pregnancy-related care cannot be optimized for these women. Thus, substantially decreasing the median gestation at presentation for ANC and abortion is critical to improving maternal and child health in Nigeria and other similar settings.

Late presentation for pregnancy care is a well-documented and persistent problem in sub-Saharan Africa [7]. In this study, the majority of women attending ANC and TOP services had heard of urine pregnancy testing as a means of confirmation of pregnancy. However, few women were aware that a pregnancy could be detected by a urine test one to two weeks after a missed period. 
An additional concern is that the majority of ANC clients (61\%) thought it was appropriate to make the first ANC visit after the first trimester of pregnancy.

This study has demonstrated that obtaining a urine pregnancy test at a pharmacy is associated with an earlier presentation for ANC and abortion services. Greater access to private sector urine pregnancy testing alone, however, is not likely to decrease gestational age at presentation sufficiently, as a significant proportion of pregnant women utilize public sector health services. Hence, there is a need to make available and accessible urine pregnancy testing in public health facilities that provide pregnancy care-related health services. Making urine pregnancy testing routinely available in public and private clinics and combining testing with initiation of care at point of confirming pregnancy or immediate referral to appropriate pregnancy care has the potential to significantly decrease gestational age at first visit. The establishment of a pregnancy confirmation clinic, using urine pregnancy testing, at selected antenatal clinics in an urban area of South Africa led to, on average, a 10-week decrease in the gestational age of booking for antenatal care [8].

\subsection{Limitations}

There are several limitations in this study. The participants had all reached pregnancy care in the private sector and were making a first visit for either ANC or abortion services; there is no data on women who never reached or did return to the private-sector services after being sent away-for example, women seeking abortion who were so delayed that they were denied a safe abortion were not included in this study. In addition, this quantitative survey is unable to shed light on complex patientrelated factors for late presentation. Qualitative methodologies which will be more informative are required.

\subsection{Recommendations}

There are a few recommendations that arise from the findings of this study and specific programmes designed from these, may be applicable in diverse healthcare settings. Firstly, this study identified some deficit in client knowledge about pregnancy recognition and care. This misinformation on the part of women requires urgent correction through community-based and health service-based education about the importance of early pregnancy recognition and presentation for care. Public awareness campaigns about these issues should be undertaken in general community settings and in all clinics that serve female clients, not only in ANC and abortion services. In addition, this study documented a lack of health education materials, which inform women about the importance of early presentation for care, and of the services available. There is a need to develop such materials.

Secondly, there is a need to arm the providers with clear protocols and guidelines for urine pregnancy testing. These protocols should clarify the following: that urine pregnancy testing is an on-site health service, when pregnancy tests should be done and by whom, and the appropriate referrals for and management of clients with positive and negative pregnancy test results.

Thirdly, a significant number of providers held negative views about conducting pregnancy testing for teenage clients and about promoting pregnancy testing more generally. These findings indicate the need for values clarification workshops with providers so that they are able to understand the individual, public health, and health care resource-related benefits of early pregnancy confirmation and are, thus, better equipped to provide pregnancy testing services to all clients irrespective of their personal views.

Fourthly, a substantial proportion of women who are ultimately cared for in the private sector confirm their pregnancy in the private pharmacies separate from the clinics. There is need for better interaction between the private clinics and private pharmacies in order to ensure that these women receive optimal and timely care. Health program managers need to inform the private sector providers of the problem of late pregnancy confirmation and of the appropriate public and private sector referral routes if test results are positive or negative.

Finally, health care providers mentioned concerns that if pregnancy testing is promoted, health sector services will be overwhelmed by clients requesting pregnancy tests. Clients cited long clinic waits as a barrier to obtaining pregnancy tests. Therefore, from both health services and client perspectives, these findings support the need for establishment and advertisement of "fast-track" pregnancy confirmation services in public and private clinics. These services should enable women to bypass the usual clinic delay and quickly obtain a urine pregnancy test conducted by non-professional members of staff (e.g. a community health worker or lay counselors). The testing should be combined with either immediate initiation of care or referral to appropriate pregnancy care, depending on the range of services available at a particular clinic.

\section{CONCLUSION}

Bearing in mind that the clinical benefit and public health impact of early presentation for antenatal and abortion services are unequivocal, strategies aimed at decreasing the gestational age at presentation for pregnancy care, such as the increased use of urine pregnancy testing, should be given priority. Urine pregnancy testing 
is inexpensive, not staff intensive and logistically simple to implement. The implementation of urine pregnancy testing services has the potential to improve both maternal and neonatal health by decreasing gestational age at presentation for ANC and abortion services among women who are pregnant, as well as by channeling nonpregnant women who wish not to become pregnant into contraceptive care.

\section{ACKNOWLEDGEMENTS}

The author wishes to express his gratitude and appreciation to all the interviewers and respondents who participated in this study.

\section{REFERENCES}

[1] Bastian, L.A. and Piscitelli, J.T. (1997) Is this patient pregnant? Can you reliably rule in or out early pregnancy by clinical examination? The Journal of the American Medical Association, 278, 586-591. doi:10.1001/jama.1997.03550070078042

[2] World Health Organisation (2006) Making pregnancy safer: Strategic approach to improving maternal and newborn survival and health. Geneva.

[3] World Health Organisation (2004) Safe abortion: Technical and policy guidance for health systems. Geneva.

[4] Stanback, J., Raymond, E. and Janowitz, B. (2002) Hor- monal pregnancy test redux. Contraception, 66, 295-296. doi:10.1016/S0010-7824(02)00363-3

[5] Wilcox, A., Bairs, D.D., Dunson, D., McChesney, R. and Weinberg, C. (2001) Natural limits of pregnancy testing in relation to the expected menstrual period. The Journal of the American Medical Association, 286, 1759-1761. doi:10.1001/jama.286.14.1759

[6] Lamina, M.A. (2004) Gestational age at first antenatal attendance in Sagamu, Western Nigeria. Nigerian Journal of Clinical Practice, 7, 1-3.

[7] Myer, L. and Harrison, A. (2003) Why do women seek antenatal care late? Perspectives from rural South Africa. Journal of Midwifery and Women's Health, 48, 268-272. doi:10.1016/S1526-9523(02)00421-X

[8] Jeffery, B.S., Tsuari, M., Pistorius, L.R., Makin, J. and Pattison, R.C. (2000) The impact of a pregnancy confirmation clinic on the commencement of antenatal care. South African Medical Journal, 90, 1563-1566.

[9] Morroni, C. and Moodley, J. (2006) The role of urine pregnancy testing in facilitating access to antenatal care and abortion services in South Africa: A cross-sectional study. BMC Pregnancy and Childbirth, 6, 28. doi:10.1186/1471-2393-6-26

[10] Burnhill, M.S. (1986) Reducing the risks of pregnancy termination. In: Landy, U. and Ratnam, S.S., Eds., Prevention and Treatment of Contraceptive Failure, Plenum, New York, 141-147. doi:10.1007/978-1-4684-5248-8_21 